\title{
Cognitive impairment in patients with atrial fibrillation: What is the importance of anticoagulant therapy?
}

\author{
Giovanni Capobianco
}

\author{
“Transmurale" Geriatric Unit, S. Eugenio Asl Roma 2 Hospital, Rome, Italy
}

\begin{abstract}
Atrial fibrillation represents one of the most significant aging markers and its prevalence increases with age, reaching $11.1 \%$ in men and $9.1 \%$ in women aged over 85 years.

Fragility is nowadays defined according to two different phenotypes: the biologic-physiologic phenotype developed by L. Fried and Baltimora School and the clinic-multidimensional phenotype, proposed by Rockwood and Halifax School.

If the clinical characteristics of elder patients hospitalized in acute geriatric unit care are considered, differences appear in the clinical features, in co-morbidity, in therapy and prognosis.
\end{abstract}

\section{Introduction}

The most frequent clinical features, according to my personal records, are represented by a sudden or gradual global functional loss (BADL, dispnea, delirium, worsening of an already existent cognitive impairment and postural instability, with possible falls).

In fragile elder people, atrial fibrillation is associated to several comorbidities; well known clinical consequences are represented by mortality increase and by cardio-embolic and silent stroke risk increase.

Dementia risk is also increased in patients with "stroke-free" atrial fibrillation and clinical studies have demonstrated the association among atrial fibrillation, cognitive impairment and hippocampal atrophy, as highlighted by neurological investigations [1].

Corresponding author: Giovanni Capobianco, UOC "Transmurale", Ospedale S. Eugenio, Asl Roma 2, Rome, Italy.

E-mail: capobiancog58@gmail.com

Key words: Atrial fibrillation; dementia; warfarin; DOACs.

Received for publication: 24 April 2018

Accepted for publication: 30 May 2018

(C) Copyright G. Capobianco, 2018

Tipografia PI-ME Editrice, Italy

Monaldi Archives for Chest Disease 2018; 88:956

doi: 10.4081/monaldi.2018.956

This article is distributed under the terms of the Creative Commons Attribution Noncommercial License (by-nc 4.0) which permits any noncommercial use, distribution, and reproduction in any medium, provided the original author(s) and source are credited.
There is similarity among the scores obtained through MMSE in medial temporal lobe atrophy and with cerebral white matter hyperintensity.

The pathogenetic mechanisms causing the increased prevalence of dementia in patients with atrial fibrillation are several, such as thromboembolism and stroke, microhemorrhages caused by anticoagulants, cerebral hypoperfusion, inflammatory mechanisms and, probably, other not yet known causes.

It seems that ventricular rate control influences cognitive tests scores, which are compromised if the ventricular rate test is stable under $60 \mathrm{bpm}$ or above $90 \mathrm{bpm}$. As of dementia prevalence, any difference has been registered when using rhythm or rate control strategies.

It has been demonstrated that atrial fibrillation is associated to cognitive impairment regardless of stroke or other cardiovascular risk factors and the presence of cognitive impairments is doubled in patients with atrial fibrillation, reaching $40-50 \%$ higher risk of dementia, Alzheimer and others, regardless of stroke, in patients with atrial fibrillation.

The anticoagulant therapy is a fundamental tool in cardio-embolic risk prevention: to warfarin traditional therapy, it has now been added a direct anticoagulant therapy using different molecules grouped under the name of DOACs.

\section{Definition of DOACs}

Since a number of years, attention is focused on the role of the anticoagulant therapy in patients with atrial fibrillation moderate-high risk and on the effect on dementia end-point, by comparing DOACs and warfarin.

It is known that patients with atrial fibrillation have an increased dementia risk, which is influenced by "warfarin control". Warfarin long-term treatment exposes patients with atrial fibrillation to the risk of cerebral micro-hemorrhages, in "overtreatment" cases, or to cerebral microemboli in "undertreatment" cases; hemorrhages risk increases in those patients taking aspirin to treat a coexisting coronary disease.

Since some years, DOACs represent an extraordinary alternative to warfarin due to the predictable anticoagulant effect, to the higher compliance in patients who do not need INR dosage and to the lower risk of embolic stroke and intracranial hemorrhage in the treated patients. Recent studies have compared the effect of DOACs $v s$ warfarin on several outcomes, such as stroke, TIA and dementia [2,3]. Many studies concluded that DOACs have an advantage over warfarin in treating cerebral-vascular problems [4].

If "macrovascular" benefits (lower embolic stroke and hemorrhage risk) are transposed at "microvascular" level, DOACs have proved to reduce the risk of dementia in patients with atrial fibrillation moderate-high risk more than warfarin. Several studies have demonstrated that, in percentage, the effect on stroke/TIA/dementia combined endpoint after one year, three years and on the long term is different in patients treated with DOACs or with warfarin, with better results in the 
first group. Generally, it can be stated that long-term dementia frequency (under all causes) is lower in patients treated with DOACs than in patients treated with warfarin.

Comparison studies on different D0ACs types are now being carried on, but currently, significant differences among the several molecules have not been registered.

In conclusion, it can be stated that there is a clinically relevant association among atrial fibrillation, cognitive impairment and dementia and that one of the most important pathogenetic mechanisms is represented by clinically evident or silent embolic problems and/or by micro or micro hemorrhages [5].

In a wide group of patients with atrial fibrillation at moderate-high risk, the risk of dementia, stroke and TIA is lower in patients treated with D0ACs than in patients treated with warfarin, without significant differences with DOACs combinations.

The lower incidence of cognitive impairment and dementia in patients with atrial fibrillation treated with DOACs than in patients treated with warfarin is a further reason, in fragile elder people, to prefer these new molecules to warfarin, which had been previously used in the anticoagulant therapy.

\section{References}

1. Dublin S, Anderson ML, Haneuse SJ, et al. Atrial fibrillation and risk of dementia: Prospective cohort study. J Am Geriatr Soc 2011;59:1369-75.

2. Bunch TJ, May HT, Bair TL, et al. Atrial fibrillation patients treated with long-term warfarin anticoagulation have higher rates of all dementia types compared with patients receiving long-term warfarin for other indications. J Am Heart Assoc 2016;5. pii: e003932.

3. Jacobs V, May HT, Bair TL, et al. Dementia and stroke rates were lower among patients receiving long term anticoagulation therapy with novel oral anticoagulations compared to warfarin. Circulation 2015;132:A11919.

4. Jacobs V, May HT, Bair TL, et al. Long-term population-based cerebral ischemic event and cognitive outcomes of direct oral anticoagulants compared with warfarin among long-term anticoagulated patients for atrial fibrillation. Am J Cardiol 2016;118:210-4.

5. Ott A, Breteler MMB, de Bruyne MC, et al. Atrial fibrillation and dementia in a population-based study. The Rotterdam Study. Stroke 1997;28:316-21. 